Rappelons, en terminant, la technique de la réaction :

Le distillat de $10 \mathrm{~cm}^{3}$ renfermant le diacétyle à doser est additionné de $0 \mathrm{~cm}^{3} 5$ de solution aqueuse de chlorhydrate de diaminobenzidine à $2,5 \%$ préparée au moment de l'emploi ou quelques heures (au plus) auparavant. Agiter. Ajouter $0 \mathrm{~cm}^{3}-5$ d'acide chlorhydrique concentré. Agiter. Attendre 2 minutes. Colorimétrer au photomètre préalablement étalonné ou comparer à une gamme de teintes préparées avec des solutions de bichromate de potas. sium.

Le titrage proprement dit d'une solution de diacétyle ne demande done que quelques minutes.

Pour tous détails concernant le dosage du diacétyle dans les beurres, l'importance des prises d'essai, la conduite de la distillation, ete., nous renvoyons à notre travail antérieur (Le Lait, juilletaoût 1937 , t. XVII, pages 673 à 698 ).

\title{
INDEX COLORIMÉTRIQUE D'ACIDIFICATION DES LIPIDES DU BEURRE
}

\author{
par \\ Etienne GOIFFON \\ Ingénieur I.A.N.
}

On sait que ces corps subissent du fait des oxydations spontanées ou des lipases que leur extraction a entrainées, ou qui ont été développées par certains microbes, des transformations qui sont groupées sous le nom de Rancissement. En réalité, il faut distinguer parmi ces altérations, celles qui consistent en la formation de certains corps volatils (aldéhydes, etc., acides gras volatils), qui donnent un goût et une odeur désagréables aux corps gras alimentaires d'une part, et d'autre part, la mise en liberté des acides gras normalement liés au glycérol.

C'est ce second groupe d'altération qu'on apprécie généralement par le dosage d'acidité et qui seul fait l'objet de nos recherches.

Plusieurs méthodes ont été proposées dans ce but. La plus classique consiste à solubiliser les acides gras formés dans ees graisses et huiles, et à les doser grâce à une solution alcaline titrée en présence de phénolphtaléine comme indicateur.

Cette méthode n'est pas très compliquée. Elle demande cependant un minimum d'appareillage et de soin pour l'établissement des liqueurs titrées qui en font plus une méthode de laboratoire qu'un procédé d'appréciation utilisable par le petit industriel.

Une autre méthode toute différente a été proposée récemment 
par KNAYSI : elle se ramène à une mesure colorimétrique des variations quantitatives de coloration de la base de l'indicateur soluble dans les graisses.

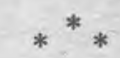

Nous avons pensé qu'il serait intéressant d'utiliser une réaction quantitative colorée qui, a fait l'objet d'une courte note de R. Golffon (Soc. de Biologie Clinique, 1948).

Quand on verse dans une solution de bleu de Nil (sulfate ou chlorure) une solution concentrée de $\mathrm{CO}^{3} \mathrm{Na}^{2}$, ce colorant vire au rouge.

Si la solution de bleu de Nil contient une trace d'acide gras non saturé tel que l'acide oléique, la coloration reste bleue malgré l'adjonction de $\mathrm{CO}^{3} \mathrm{Na}^{2}$. Si les quantités d'acide oléique sont suffisamment faibles, il se produit dans ees conditions une gamme de couleur allant du rouge au bleu en passant par toutes les teintes du violet. La quantité de couleur bleue appréciée au colorimètre à l'écran rouge est directement proportionnelle à l'acide oléique présent.

Ce phénomène n'a été observé qu'avec les acides insaturés et, chose curieuse, il ne semble pas proportionnel au nombre de doubles liaisons de l'acide gras utilisé.

D'après des travaux en cours de Mme Couchoud, un même poids moléculaire d'acide oléique, linoléique et linolénique donnent des intensités colorées qui sont dans les proportions respectives de $3,2,1$, c'est-à-dire, à l'inverse du nombre des doubles liaisons ; ce phénomène n'a pas été encore expliqué.

Les acides gras saturés employés à ce sujet ne donnent pas cette réaction (tels les acides laurique, palmitique, stéarique). L'acide butyrique notamment ne donne pas cette coloration.

Cette méthode, extrêmement sensible, a été utilisée en biologie pour apprécier la quantité d'acides gras libre du sérum, certains acides gras des matières fécales, etc.

Nous avons pensé qu'il pouvait être intéressant de transposer cette méthode dans l'industrie à cause de sa simplicité, et malgré les limites que nous venons de lui reconnaître.

\section{MÉTHODE GÉNÉRALE DE DOSAGE COLORIMÉTRIQUE DES ACIDES GRAS AU BLEU DE NIL}

Le complexe bleu de Nil-acide gras, forme une solution colloïdale stable; il ne vire pas au rouge en présence de carbonate de soude, mais vire au rouge en présence de soude. 
La formation du complexe bleu est entravée par de petites quantités d'acétone et d'alcool.

La forme rouge du colorant est peu soluble dans l'eau et sa solution se trouble rapidement.

Ces notions entraînent la nécessité d'éliminer l'alcool et l'acétone des solutions dans lesquelles se ferait la réaction. D'autre part, il serait nécessaire, puisqu'il s'agit d'un dosage photométrique, d'entraver le trouble rapidement croissant qui se produit avec là forme rouge du bleu de Nil.

C'est pourquoi nous employons une solution gommeuse povr maintenir sa transparence, suivant en cela la technique mise au point par $\mathrm{M}^{\mathrm{me}}$ Covchoud dans sa thèse pour le dosage des acides gras au moyen du bleu de Nil.

Deux corps nous ont donné de bons résultats : la gomme arabique et l'alginate de soude (1). La gomme arabique à $5 \%$ doit être traitée auparavant par une pincée d'oxalate d'ammoniaque pour préoipiter le calcium qu'elle contient en abondance et qui, dans le milieu final carbonaté, formerait un trouble gênant. L'alginate de soude ne contient pas de calcium et peut être employé directement.

La réaction type est la suivante :

Une solution d'oléate de soude est préparée avec 0 gr. $020 \%$ d'acide oléique et alcalinisée suffisamment pour le dissoudre. On en prend $4 \mathrm{~cm}^{3}$ correspondant à $0 \mathrm{mgr}$. 8 d'acide oléique qu'on place dans une éprouvette exactement jaugée à $30 \mathrm{~cm}^{3}, 3 \mathrm{~cm}^{3}$ d'alginate de soude à $0,5 \%$ et de l'eau jusqu'à $20 \mathrm{~cm}^{3}$; on mélange soigneusement; on ajoute $4 \mathrm{~cm}^{3}$ d'une solution aqueuse de bleu de Nil à 0 gr. $5 \%$; $5 \mathrm{~cm}^{3}$ de $\mathrm{CO}^{3} \mathrm{Na}^{2}$ pur cristallisé à $20 \%$ et on complète à $30 \mathrm{~cm}^{3}$ avec de l'eau distillée ; il se développe une coloration violette ; on dose le bleu qu'elle contient au photomète à l'écran rouge.

Une courbe de référence peut être construite avec des quantités croissantes d'acide oléique de 0,2 à 1 milligramme qui permettra de mesurer l'acide oléique des quantités de savon contenues dàns une solution inconnue.

Voici le graphique que nous avons obtenu avec l'appareil de Vernes écran rouge (on pourrait aussi bien utiliser tout autre photomètre pour l'établissement de la eourbe et les dosages).

On soustrait du chiffre obtenu à l'appareil la D. Optique d'un témoin fait dans les mêmes conditions, mais sans oléate.

(1) Alg-Gum. - Etablissements Maton, 16, rue de Naples, Paris. 


\section{Courbe du bleu de Nil avec les acides gras}

Sol: acide oléique alealinisé $0,020 \%$
$+5 \mathrm{~cm}^{3}$ sol. gomme oxalatée à $10 \%$

$+4 \mathrm{em}^{3}$ sol. de bleu de Nil R.A.L. $0,050 \%$

+ Eau distillée q. s. pour $25 \mathrm{~cm}^{3}$

$+5 \mathrm{~cm}^{3}$ sol. $\mathrm{CO}^{3} \mathrm{Na}^{2}$ à $20 \%$ pur eristallisé

Densités optiques (Vernes) Ecran $\alpha$

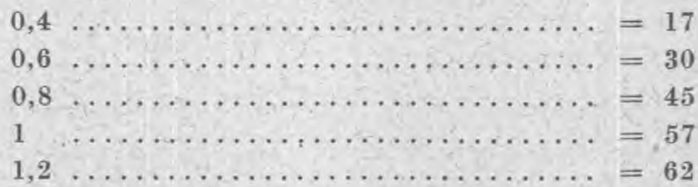

\section{Technique sur le beurre}

La technique générale consiste à extraire les acides gras du beurre et à pratiquer sur leur solution alcaline aqueuse (à l'état de savon) la réaction au bleu de Nil dans des conditions telles qu'il $\mathrm{y}$ ait un excès de bleu de Nil par rapport à la quantité de savon, et à doser colorimétriquement la quantité de bleu de Nil qui, grâce à sa combinaison avec le savon, n'a pas viré au rouge sous l'action du carbonate de soude.

\section{A. Prélèvement.}

Nous utilisons de très faibles quantités de beurre. Nous le faisons fondre à une température douce pour le deshydrater et le transvaser dans un autre récipient à l'état pur. On peut, après refroidissement, en peser 1 gramme dans une eapsule, mais la difficulté serait de placer ce gramme sans perte au fond d'un tube à essai. Si la chose était absolument nécessaire, il serait indispensable de dissoudre par l'éther ce beurre, de verser sa solution éthérée dans le tube à essai qui nous servira de laboratoire et ensuite d'évaporer cet éther.

On peut égalemeut peser directement le gramme de beurre dans un bécher de $30 \mathrm{~cm}^{3}$, suffisamment léger pour qu'une pesée soit possible.

Pour les dosages de routine, on peut porter le beurre à sa température de fusion, on prélève $1 \mathrm{~cm}^{3}$ dans une pipette réchauffée à la flamme. Cette mesure est loin d'être exacte, une quantité non négligeable de beurre adhère aux parois, mais c'est une erreur systématique qui permet des mesures comparatives.

L'expérience a montré que $1 \mathrm{~cm}^{3}$ de beurre fondu correspond à environ 0 gr. 86 de beurre. 


\section{B. Extraction alcoolique des acides gras.}

$10 \mathrm{~cm}^{3}$ d'une solution alcoolique aux environs de $85^{\circ}$ (alcool éthylique à $96^{\circ}=90 \mathrm{~cm}^{3}+10 \mathrm{~cm}^{3} \mathrm{~d}^{\prime} \mathrm{HCl} \mathrm{N} / 10$ ) sont versés sur la prise d'essai de beurre placée soit dans un tube à essai large, ou dans un petit bécher; le tout est porté à une température de 30 à $45^{\circ}$ et agité prudemment pour obtenir une division du beurre en fines gouttelettes, sans aller jusqu'à l'émulsion proprement dite. On laisse reposer un temps suffisant pour que les gouttelettes tombent au fond du tube.

De la partie supérieure on prélève $5 \mathrm{~cm}^{3}$ que l'on fait évaporer dans une capsule. On reprend le résidu dans la capsule encore chaude par quelques gouttes de carbonate de soude et quelques centimètres cubes d'eau, en frottant le fond avec un agitateur muni d'un caoutchouc. Cette solution contient tous les acides gras à l'état de savon. On la verse dans une éprouvette de $30 \mathrm{~cm}^{3}$ et e'est sur cette solution que se fera la réaction. Cette manœuvre repétée sur le même beurre par des expérimentateurs différents a fourni les mêmes valeurs finales.

\section{Dosage colorimétrique.}

La solution savonneuse, quel que soit le mode employé d'extraction, est versé dans une éprouvette de $30 \mathrm{~cm}^{3}$; on ajoute $3 \mathrm{~cm}^{3}$ d'algïnate de soude à $0,5 \%$, de l'eau jusqu'à $20 \mathrm{~cm}^{3}$; on mélange soigneusement, on ajoute ensuite $4 \mathrm{~cm}^{3}$ de la solution à $0,050 \%$ de bleu de Nil, $5 \mathrm{~cm}^{3}$ de carbonate de soude pur cristallisé à $20 \%$ et de l'eau jusqu'à $30 \mathrm{~cm}^{3}$. On mélange et, au bout de 10 minutes, on fait une mesure colorimétrique, comme nous l'avons vu plus haut. Cette mesure colorimétrique ne peut se faire que si la teinte obtenue est violacée, ce qui prouve la présence d'un excès de colorant non combiné aux acides gras, et, par conséquent, que la quantité de colorant a été plus que suffisante pour atteindre tous les acides gras présents dans la solution.

Si la coloration est bleue intense, cela laisse supposer qu'il y a trop d'acides gras pour la quantité de colorant. On ajoute alors $25 \mathrm{~cm}^{3}$ d'une solution contenant la même quantité d'alginate et de bleu de Nil, et on complète par $5 \mathrm{~cm}^{3}$ de carbonate de soude. On dilue ainsi jusqu'à ce que la solution devienne violette, et soit ainsi mesurable colorimétriquement.

On multipliera le résultat obtenu sur le graphique par le nombre de fois qu'aura été diluée la solution primitive.

Le liquide contenant les savons n'est pas toujours limpide quelles que soient les précautions prises; il se produit le plus souvent une émulsion qui provoque un léger trouble dans le liquide, ce 
trouble est mesuré par le photomètre et ne permettrait pas une mesure exacte de l'intensité colorée.

Il faut done soustraire du chiffre lu sur l'appareil un témoin dans lequel tous les éléments optiques autres que le bleu seront mesurés.

On arrive à ce résultat : en ajoutant à $5 \mathrm{~cm}^{3} \mathrm{du}$ liquide une goutte de lessive de soude à $36^{\circ} \mathrm{B}$, qui fait virer la coloration en rouge.

\section{Résultats obtenus.}

Nous avons fait des dosages comparés entre l'acidité de titration à la potasse alcoolique et notre indice de bleu dans différents beurres, les uns frais, les autres plus ou moins rances.

Certains dosages ont été répétés deux ou trois fois sur le même échantillon et ont fourni alors des chiffres comparables les uns aux autres et de même ordre de grandeur. Avec la même technique et à la même époque, avec les mêmes solutions, des beurres différents nous ont donné des proportions très différentes entre l'acidité de titration et l'indice de bleu, tous nos résultats étaient exprimés en acide oléique.

Par exemple l'indice de bleu nous indiquant 2 grammes d'acide oléiquepar kilogramme, l'acidité de titration indique 24 gr. 80 .

Il est permis d'en conclure que l'acide oléique ne représente qu'une très faible partie des acides libérés par oxydation ou rancissement. L'absence de rapport constant entre ces deux données montre que certains beurres développent une acidité (butyrique par exemple) beaucoup plus forte ou beaucoup plus faible que certains autres beurres, à égalité de libération d'acide oléique.

Le comportement du rancissement du beurre n'est pas du tout le même que celui de l'hydrolyse des huiles, dans ee dernier cas il s'agit surtout d'une action lipasique qui nous a donné une correspondance beaucoup plus régulière entre l'acidité de titration et l'indice de bleu de Nil, traduisant les acides insaturés libérés.

Il eut été intéressant d'établir une relation entre l'indice d'iode des acides gras libres du beurre et la réaction au bleu de Nil, sensible, elle aussi, aux liaisons éthyléniques. Mais les très petites quantités d'acides gras libres que comporte le beurre, ne permettent pas d'effectuer avec sécurité la détermination de l'indice d'iode.

$$
*^{*} *
$$

Nous avons établi cette méthode parce qu'il est nécessaire de partir d'une technique rigoureuse avant d'en faire des applications 
DOSAGE DES AGIDES GRAS INSATURÉS LIBRES (exprimés en acide oléique)

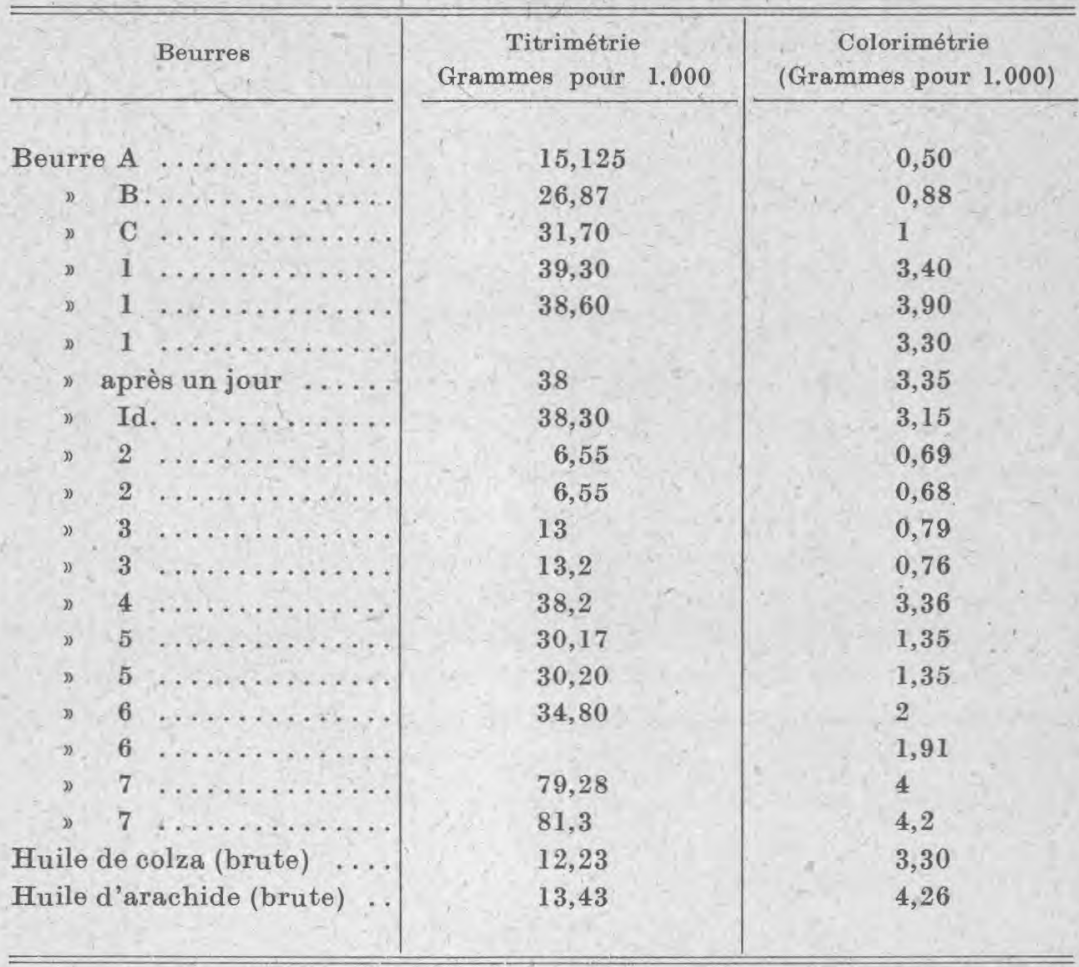

approchées ; il est douteux, en effet, qu'elle supplante la méthode de titrimétrie habituelle qui ne demande pas d'appareils photométriques, bien que nous indiquions une méthode titrimétrique pour le bleu de Nil, susceptible d'une certaine précision.

En réalité, comme nous l'avons vu, la mesure titrimétrique et notre dosage colorimétrique peuvent ne pas coïncider, la réaction au bleu de Nil, ne traduisant que la présence d'acides libres non saturés, les acides saturés leur échappant complètement.

A un point de vue plus scientifique, ce procédé permet de suivre le dédoublement sur de très petites quantités d'un corps gras, ce qui peut rendre service pour les recherches expérimentales.

Si nous avons cherché à obtenir des mesures rigoureuses de l'indice du bleu de Nil, e'était surtout pour glisser avec sécurité vers des méthodes de plus en plus pratiques qui puissent rendre service aux industriels.

Nous présenterons donc maintenant des méthodes qui mèneront à des approximations de plus en plus larges, qui permettent au 
contre-maître de se rendre compte de l'évolution vers l'acidité d'un beurre.

\section{Première méthode.}

Emploi d'un étalon coloré, fixe et titrimétrie, jusqu'à égalité avec cet étalon coloré.

\section{Deuxième méthode.}

De large orientation.

Réaction sans extraction préalable.

\section{MÉTHODE SIMPLE D'APPRÉCIATION PRATIQUE}

Le principe de ce procédé consiste à ajouter à la solution contenant des acides gras, du carbonate de soude et des-affusions successives d'un bleu de Nil titré jusqu'à l'apparition de la forme rose alcaline que prend le colorant quand il n'est pas combiné aux acides gras.

Cette apparition se signale par une teinte violette que l'on compare à un étalon coloré artificiel de composition chimique défini. Le pouvoir de combinaison de la solution de bleu de Nil a été préalablement étalonné grâce à une solution titrée d'oléate de soude.

$$
*^{*} *
$$

\section{Technique générale}

10 Etalon coloré.

Dans une éprouvette de $30 \mathrm{~cm}^{3}$, on verse successivement $5 \mathrm{~cm}^{3}$ de la solution suivante :

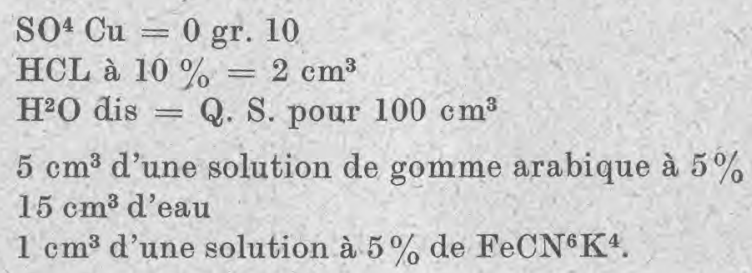

On mélange après chaque affusion; il se produit une coloration rouge de férrocyanure de cuivre.

On ajoute ensuite 1 ou $2 \mathrm{~cm}^{3}$ de la solution de bleu de Nil à $0,5 \%$. On complète à $30 \mathrm{~cm}^{3}$; on obtient un mélange transparent violet ;

20 Solution d'acide oléique, $0 \mathrm{gr}$. $2 \%$, préparée comme précédemment; 
$3^{\circ}$ Solution de bleu de Nil (sulfate ou chlorure à $0,6 \%$ ) ;

$4^{\circ}$ Alcool éthylique à $85^{\circ}$, contenant $H C L \quad N / 10=10 \mathrm{~cm}^{3} \%$;

$5^{\circ}$ Solution d'alginate de soude à $0,5 \%$.

\section{Titration du bleu de $\mathrm{Nil}$}

On place dans une éprouvette de $30 \mathrm{~cm}^{3}, 4 \mathrm{~cm}^{3}$ de solution d'oléate à $0,2 \%$ d'acide oléique ; on ajoute $15 \mathrm{~cm}^{3}$ d'eau et $5 \mathrm{~cm}^{3}$ d'alginate à $0,5 \%$.

Mélanger soigneusement.

A l'aide d'une burette on laisse tomber, en mélangeant à chaque fois la solution titrée de bleu de Nil, jusqu'à ce que la coloration, d'abord bleue, devienne violette, tendant à une teinte semblable au mélange cuivrique étalon. On attend quelques minutes que la coloration, qui tend à bleuir, se stabilise en la maintenant par des affusions légères de bleu de Nil, dont l'excès donnera une coloration rouge dans la solution carbonatée.

On note le volume employé. Ce volume représente, dans les conditions de l'expérience, 0 mgr. 8 d'acide oléique.

\section{Titration d'un corps gras}

Pour cela, on pratique l'extraction alcoolique de beurre comme nous l'avons indiqué dans la première partie de ce travail. On évapore l'alcool, on reprend par le carbonate de soude et on lave la capsule par de l'eau carbonatée qu'on amène à un volume de $10 \mathrm{~cm}^{3}$.

On en prélève $5 \mathrm{~cm}^{3}$ qu'on verse dans une éprouvette de $30 \mathrm{~cm}^{3}$; on ajoute alginate, carbonate de soude et eau comme précédem. ment, et on titre avec la solution de bleu de Nil jusqu'à obtention d'un violet analogue à celui de l'étalon au cuivre.

On fera aisément le calcul d'après la formule suivante :

$$
\frac{0 \mathrm{mgr} .8 \times \mathrm{V}}{\mathrm{V}^{\prime}}
$$

V: Volume employé pour l'égalité de teinte.

$V^{\prime}$ : Volume employé pour amener l'oléate de soude au virage voulu.

Pour les acides gras contenus dans 0 gr. 50 de beurre, il faudra multiplier le chiffre obtenu par 500, pour obtenir la quantité d'acides dosés dans 1: kilogramme de beurre, exprimé en acide oléique.

Ce procédé n'offre que peu de difficultés de lecture, malgré la présence du trouble formé par les globules de graisse neutre entraînés quand le beurre ne contient pas plus de 1 gr. 6 pour 1.000 d'acides libres. 
Si, malgré l'affusion de quantité de bleu dépassant le double ou le triple du volume utilisé pour l'étalon, l'appréciation devient extrêmement difficile étant donné l'intensité de la couluer, on aura avantage, dans ce cas, à recommencer la mesure en utilisant, par exemple, $1 \mathrm{~cm}^{3}$ du liquide d'extraction restant, puisque nous n'en aurons prélevé que $5 \mathrm{~cm}^{3}$.

Il faudra tenir compte dans les calculs du volume de cette nouvelle prise d'essai.

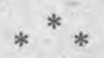

\section{Technique plus simplifiée}

Elle consiste à faire la réaction directement sur le corps gras sans extraction préalable.

On met $1 \mathrm{~cm}^{3}$ de beurre, ou moins encore, dans une éprouvette de $30 \mathrm{em}^{3}$ en ajoutant simplement $5 \mathrm{~cm}^{3}$ de $\mathrm{CO}^{3} \mathrm{Na}^{2}$ pur eristallisé à $20 \%$ et de l'eau jusqu'à $25 \mathrm{~cm}^{3}$. Une émulsion se forme qu'on favorise par agitation. On ajoute du bleu de Nil jusqu'à ce que la coloration violette apparaisse.

Avec ce procédé deux précautions sont à prendre :

$1^{\circ} \mathrm{Le}$ beurre fondu a une viscosité telle qu'il est impossible d'en mesurer exactement $1 \mathrm{~cm}^{3}$ à la pipette. Aussi employons-nous le procédé suivant :

On prélève $1 \mathrm{~cm}^{3}$ de beurre avec une pipette légèrement chauffée ; on l'égoutte soigneusement après avoir essuyé sa partie extérieure. On prélève ensuite, d'une petite quantité d'éther placée dans un tube à essai, $1 \mathrm{~cm}^{3}$ avec la môme pipette; on le mélange au beurre déjà déposé dàns un tube à essai.

C'est de cette solution éthérée-huileuse qu'on prélève très aisément $1 \mathrm{~cm}^{3}$ pour pratiquer la réaction.

Le $1 / 2 \mathrm{em}^{3}$ d'éther introduit se dissoudra aisément dans les $30 \mathrm{~cm}^{3}$ d'eau employés, et les graisses acides du $p \mathrm{H}$ de la solution carbonatée forment des savons qui passent dans l'eau.

$2^{\circ}$ Il faut projeter rapidement le bleu de Nil dans le mélange avant que ce colorant ne soit absorbé par les globules gras, qu'il colore en rouge orangé, mais au bout d'un certain temps de contact.

Si le mélange absorbe tróp de colorant avant de virer au violet, il faut recommencer la mesure avec $1 \mathrm{~cm}^{3}$ de la solution huileuseéthérée, qu'on aura mis de côté dans un tube à essai étroit, et bouché.

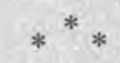




\section{Autre technique (réaction qualitative)}

On pourra utiliser cette méthode avec profit pour savoir immédiatement si un beurre contient, ou non, des acides gras en quantité anormale, en laissant tomber un fragment de beurre dans un tube à essai, en agitant avec quelques centimètres cubes de $\mathrm{CO}^{3} \mathrm{Na}^{2}$ à $20 \%$, de l'eau à peu près jusqu'à $20 \mathrm{~cm}^{3}$ et $2 \mathrm{~cm}^{3}$ environ de bleu de Nil. Un beurre normal donne une réaction rouge.

La présence d'acides gras anormale donne une réaction bleu intense.

$$
* * *
$$

\section{Conclusions}

Nous avons montré que le bleu de Nil qui vire au rouge en présence de carbonate de soude, reste bleu en présence de certains acides gras non saturés. Il se forme une combinaison du colorant et de l'acide, quantitative en se plaçant dans certaines conditions. On péut se servir de la réaction pour un dosage colorimétrique de ces acides.

Nous avons indiqué les simplifications successives de cette méthode pouvant être appliquées avec des difficultés de moins en moins grandes, jusqu'à aboutir à une réaction colorée extrêmement simple, permettant de déceler immédiatement la présence anormale d'acides gras dans un corps gras alimentaire.

\section{BIBLIOGRAPHIE}

Mme M. Couchoud. Thèse en Pharmacie (en préparation).

R. Golffon. Une réaction colorée des acides gras supérieurs. Annales de Biol. Clinique, n ${ }^{\circ} 5,1948$, p. 282.

KNaysi. Nouvelles études pour l'usage des colorants basiques pour mesurer les acides gras. Journal of Dairy Science, 1942, vol. XXV, no 7, 585.

Krakowski et KNAYst. Nouvelle méthode colorimétrique de dosage des acides gras du beurre. Journal of Dairy Seience, 1942, vol. XXV, no 8 , 651 . 